\title{
Wave Power Density Hotspot Distribution and Correlation Pattern Exploration in the Gulf of Mexico
}

\author{
Chengcheng $\mathrm{Gu}$ and $\mathrm{Hua} \mathrm{Li}$ *
}

Citation: Gu, C.; Li, H. Wave Power Density Hotspot Distribution and Correlation Pattern Exploration in the Gulf of Mexico. Sustainability 2022, 14, 1158. https://doi.org/ $10.3390 /$ su14031158

Academic Editors:

Alessandro Franco and

Tomonobu Senjyu

Received: 27 December 2021

Accepted: 18 January 2022

Published: 20 January 2022

Publisher's Note: MDPI stays neutral with regard to jurisdictional claims in published maps and institutional affiliations.

Copyright: (C) 2022 by the authors. Licensee MDPI, Basel, Switzerland. This article is an open access article distributed under the terms and conditions of the Creative Commons Attribution (CC BY) license (https:// creativecommons.org/licenses/by/ $4.0 /)$.
Mechanical and Industrial Engineering Department, Texas A\&M University-Kingsville, Kingsville, TX 78363, USA; cheng124158321@gmail.com

* Correspondence: hua.li@tamuk.edu; Tel.: +1-361-593-4057

\begin{abstract}
Wave energy has been studied and explored because of its enormous potential to supply electricity for human activities. However, the uncertainty of its spatial and temporal variations increases the difficulty of harvesting wave energy commercially. There are no large-scale wave converters in commercial operation yet. A thorough understanding of wave energy dynamic behaviors will definitely contribute to the acceleration of wave energy harvesting. In this paper, about 40 years of meteorological data from the Gulf of Mexico were obtained, visualized, and analyzed to reveal the wave power density hotspot distribution pattern, and its correlation with ocean surface water temperatures and salinities. The collected geospatial data were first visualized in MATLAB. The visualized data were analyzed using the deep learning method to identify the wave power density hotspots in the Gulf of Mexico. By adjusting the temporal and spatial resolutions of the different datasets, the correlations between the number of hotspots and their strength levels and the surface temperatures and salinities are revealed. The $R$ value of the correlation between the wave power density hotspots and the salinity changes from -0.371 to -0.885 in a negative direction, and from 0.219 to 0.771 in a positive direction. For the sea surface temperatures, the $R$ values range from -0.474 to 0.393 . Certain areas within the Gulf of Mexico show relatively strong correlations, which may be useful for predicting the wave energy behavior and change patterns.
\end{abstract}

Keywords: wave power density; correlation analysis; deep learning

\section{Introduction}

Renewable energy is becoming more and more important among different types of energy resources in U.S. electricity generation. In the United States, the potential available wave energy is about $80 \%$ of the hydrokinetic ocean energy, including currents and tidal waves [1]. The U.S. Department of Energy and the U.S Energy Information Administration estimate that the theoretical wave energy potential along the continental shelf edge can reach up to $2.64 \mathrm{TW} /$ year [2]. The United States is establishing the first wave energy test site in federal waters off the west coast of the United States, which is the PacWave South project [1]. Meanwhile, wind energy provided $8.4 \%$ of U.S. electricity generation in 2020 , an increase from $7.4 \%$ in 2019 , which is expected to continue to reach around $10.3 \%$, as forecasted by the EIA [3]. Solar energy also provided about $2.3 \%$ of the U.S. electricity generation in 2020 . The total percentage from renewable energy reached $20 \%$ of all of the U.S. electricity generation in 2020. However, possessed with the enormous potential to cover the present and future electricity needs of humans among the available ocean resources, wave energy has not reached mature commercial utilization because of its high uncertainty and its variations in both the spatial and temporal scales. Since 1890, the commercial utilization attempts have not ceased [4]. Although wave energy harvesting applications and installations have started to move from the research phase to the testing and production phases, large-scale wave energy harvesting has not been considered economically viable because of the modest output. 
Many solutions were put into practice and tested in order to attain at least the same output levels as wind and solar energy. However, the challenges related to the wide implementation of wave energy are still causing many issues, such as low utilization efficiency, the poor reliability of energy devices, and greater variations. The wave energy in the United States is rich along the west coast, with up to $240 \mathrm{TWh} /$ year, compared with the $84 \mathrm{TWh} /$ year in the Gulf of Mexico (GoM). The GoM has been classified as a low baseline area [5]. However, the activities in and along the Gulf of Mexico are thriving, with a considerable number of oil and gas platforms operating in this area. Wave energy can provide a critical portion of clean power as a substitute for inefficient gas turbines. The task of wave energy converters is to capture as much potentially available wave energy as possible in an effective way, and wave power density is one of the factors for directly assessing the potential and available wave energy in a given region. Previous research shows that the distribution of the wave power density in the GoM can provide $10 \%$ of the electricity needs of the oil platforms [2]. Although the average wave power density is less than $20 \mathrm{~kW} / \mathrm{m}$, its peak can also reach $100 \mathrm{~kW} / \mathrm{m}$ [6]. As extremely high wave heights may damage wave energy converters, it is important to predict wave energy behaviors in order to ensure an effective harvesting process.

Previous research has focused on the correlations between wave heights, wave periods, and time series [7-9]. Several potential parameters, including wind, bathymetry, salinity, and ocean surface temperature, could be improved or explored for an understanding of wave energy behaviors, which may have a critical impact on wave energy harvesting, installation site identification, wave energy converter design and maintenance, as well as the optimization of wave energy farm layouts. Exploring the wave energy behaviors introduces the definition of a wave power density hotspot, which can be defined as a geographical region with minimum wave power densities above certain thresholds during a period of time. Currently, there are no clear definitions for the ranges, duration times, and strengths of the wave power density hotspots. The wave power density hotspot has the segmentation threshold on wave power, depending on the estimating wave energy converter, the location, the wave height, and the wave period. In order to identify the range of wave power densities, a statistical analysis on the specific area is necessary. A wave power density hotspot can be identified on the basis of the value of the wave power density over a certain threshold, over a period of time, and in a predefined location.

Moreover, the ocean surface temperature and the sea surface salinity are the typical variables in physical ocean research. They are also important analysis indicators in marine research and are used for describing the basic properties of seawater. The temperature of seawater is normally fixed at the sea surface by heat exchange, where the heat is transferred from low to high latitudes by winds and the waves in the ocean. Previous research indicates that variations in the ocean surface temperature, evaporation and precipitation, and different latitudes affect ocean waves globally [10]. The changes in the surface temperature may affect wave generation and harvesting. Salinity, along with the changing ocean temperature, can affect the density of seawater, and is also a conservative tracer [11,12], which can probe the water cycle in greater detail. In addition, changes in the density field affect the wave and current generation. Hence, correlations with salinity and temperature could be explored in order to support wave energy prediction. It can be assumed that the identified wave power density hotspot distribution in the GoM may be correlated with the ocean surface temperatures and the sea surface salinities. Since wave energy behavior forecasting is not as easy as predicting the possibilities of wave movements, the correlations between wave power density hotspots and any related factors will not only provide feedback on wave energy harvesting, but will also support and enhance a deep understanding of the generation, prediction, and forecasting of wave energy, as well as of deeper scenarios and applications in the ocean.

This paper focuses on the identification and classification of a 40-year wave power density hotspot distribution, and on exploring the correlation pattern between the ocean surface temperature and the sea surface salinity. The paper identifies the number of wave 
power density hotspots and their strength levels between 1979 and 2019, and explores and analyzes, from a new perspective, the monthly, seasonal, and annual correlations of the sea surface temperatures and salinities of wave power density hotspots, using the Pearson correlation coefficient. The meteorological data from WAVEWATCH III were visualized as images of the wave power densities in 3-h time increments, which were used for wave power density hotspot identification, classification, and localization through a deep convolutional neural network. With the distribution of 40 years of wave power density hotspots, with the realistic coordinates attached and GIS mapping support, it was impossible to explore the correlations on the basis of the pattern recognition of the sea surface temperatures and salinities.

\section{Methods}

In this paper, data from different databases were used for the calculation, visualization, and analysis. In order to compare data from different databases, spatial and temporal resolutions were modified to be consistent for analyzing the correlations between different types of data. A deep convolutional neural network was used to identify the wave power density hotspots within the visualized data.

\subsection{Wave Energy Density Calculation and Visualization}

The wave power densities were calculated from 40 years (1979-2019) of meteorological data from WAVEWATCH III in the Gulf of Mexico, located at $18-30^{\circ} \mathrm{N}$ and $80-98^{\circ} \mathrm{W}$. The significant wave heights and dominant wave periods in 3-h time increments were used to calculate the wave power densities (Equation (1)). The third generation of the WAVEWATCH III system [13], operated by the National Oceanic and Atmospheric Administration (NOAA), provided the meteorological data for the waves, winds, sea surface temperatures, and other parameters, with the spatial resolution of one sixth for both the longitude and latitude.

$$
P=\frac{\rho g^{2}}{64 \pi} H_{s}^{2} T_{e} \approx\left(0.5 \frac{\mathrm{kW}}{\mathrm{m}^{3} \cdot \mathrm{s}}\right) H_{s}^{2} \mathrm{~T}_{e}
$$

where $P$ is the wave power density per unit of wave-crest length; $H_{S}$ is the significant wave height in meters; $T_{e}$ is the wave energy period in seconds; $\rho$ is the water density; and $g$ is the acceleration by gravity.

In order to visualize the wave energy density data, one of the methods is to plot the WAVEWATCH III data on a three-dimensional graph, considering the $x$-axis as the time steps, the $y$-axis as the latitude, and the $z$-axis as the longitude, to represent the data monthly, annually, or daily using MATLAB. In order to visualize the 3-h data, the toolbox based on the open-source Generic Mapping Tools was used for the manipulation of the geographic datasets through the application of the m.map mapping package. The color bar was added to indicate the different value classes of wave densities, wave heights, and wave periods. On the basis of the different color display method, the color data shows a gradual change from dark to bright for the lowest classes to the highest classes in the figure. The color bar of the visualized images was fixed at $65 \mathrm{~kW} / \mathrm{m}$ for the entire dataset as an upper threshold in order to cover the available and distinct output. The unified color bar was proven to be necessary for, and critical to, the wave density hotspot recognition. Figure 1 shows the wave-height and wave-period visualized images in different formats. There are other methods that can be used to plot the 3-h geographic data, such as ArcMap or QGIS. The authors selected MATLAB since it is easier to integrate the visualization process with the deep learning model in the same software.

\subsection{Ocean Surface Temperature and Sea Surface Salinity}

With a spatial resolution of $1 / 2$ of a degree, the ocean surface temperature dataset of the Gulf of Mexico was collected from the NCEP Climate Forecast System Reanalysis and Reforecast (CFSRR). Since the original ocean surface temperature data have different temporal resolutions, ranging from three-minute median filtered values to one-hour point 
values, in order to compare and match the temporal and spatial resolutions of the wave power density hotspot distribution during the 40-year period, the average monthly and annual temperatures were visualized. Average data were used to maintain the same 3-h time increments and were visualized as shown in Figure 2.
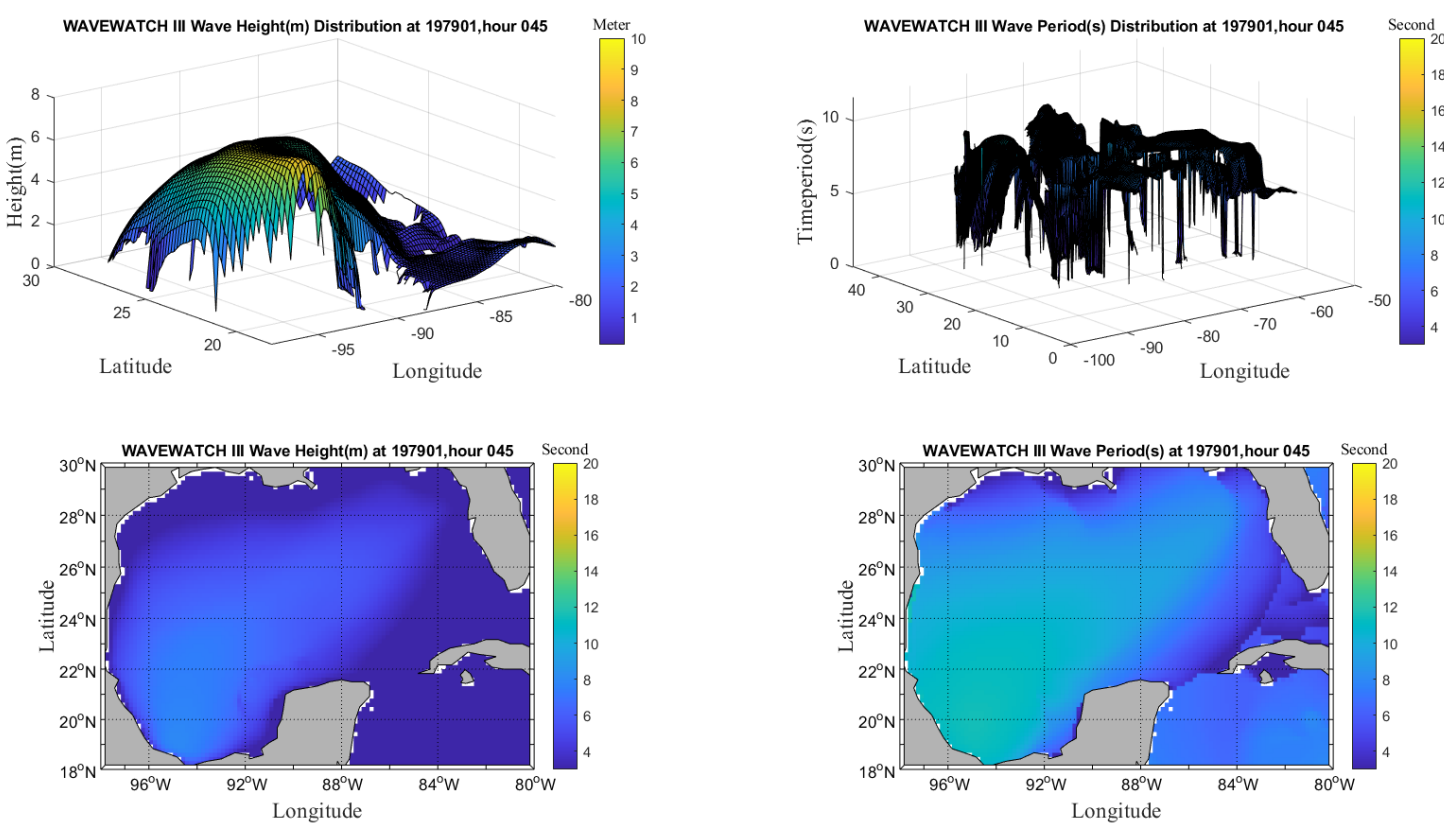

Figure 1. Visualized wave height (m) and wave period (s) distributions, at 3:00 p.m. on 1 January 1979 , in different formats.

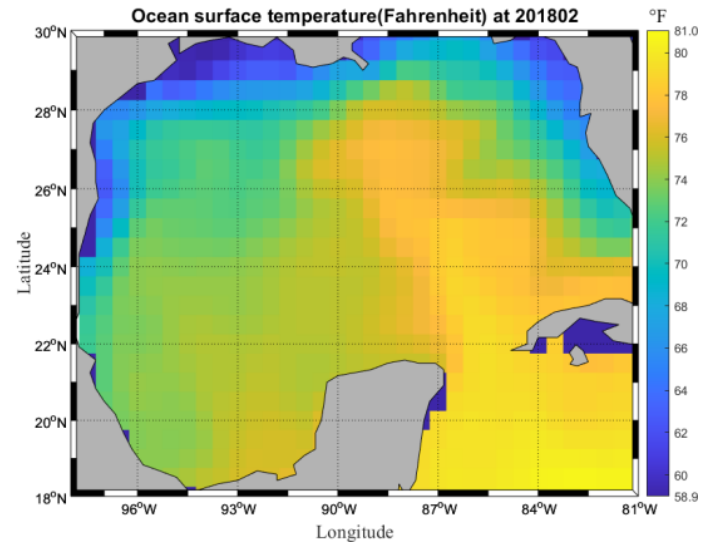

(a)

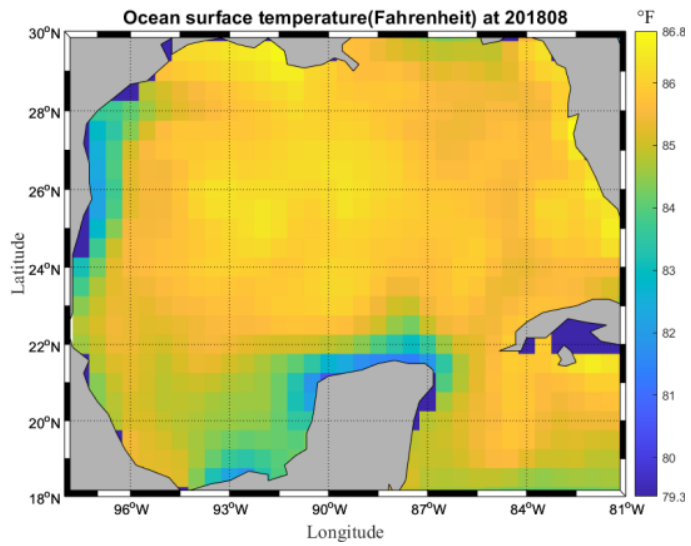

(b)

Figure 2. Surface temperature contours in the Gulf of Mexico: (a) February 2018; and (b) August 2018.

The sea surface salinity data were obtained from the NOAA near the real-time Soil Moisture Active Passive (SMAP) [14]. CoastWatch provided a 1/4-degree longitude/latitude Level 3 gridded sea surface salinity (SSS) daily mean dataset from SWAP satellite observations over the global ocean. Three-day data of a 1/4-degree resolution were used, together with the Level 3 sea surface salinity data generated from the NASA Jet Production Laboratory. Salinity data were available from 2011 to 2019, and were used to analyze the colorations of the wave power density hotspot distribution. The sea surface salinity was measured in PSUs (practical salinity units), which are regarded as the properties of conductivity and are equal to a gram per kilogram $(\mathrm{g} / \mathrm{kg})$. The average value of the global salinity is 35.5 PSU, slightly varied from 32-39 PSU in the Gulf of Mexico, and 15-40 PSU globally. 
To compare all these data in different spatial resolutions, the data were visualized and analyzed, within one degree of latitude and longitude, by comparing the gridded data, in order to comply with the same temporal and spatial values for the wave heights, wave periods, surface temperatures, and salinity resolutions. As there is no clearly defined threshold when identifying a wave power hotspot, it is challenging to use the raw gridded data from WAVEWATCH III directly. Using a CNN to analyze images does not require the definition of a specific threshold when identifying a hotspot, which makes the entire process feasible.

\subsection{Deep Convolutional Neural Network}

The structure of a deep convolutional neural network is associated with image recognition, where the feature learns to eliminate the need for handcrafted features. Feature extraction undergoes three approaches to improvement and development: (1) R-CNNs $[15,16]$ (regions with convolutional neural network features) extract up to $2 \mathrm{k}$ region proposals as candidates for the target locations, searching the whole image, pixel by pixel; (2) Fast $\mathrm{R}-\mathrm{CNN}$ s use a selective search to propose a sample hypothesis by defining the overlap threshold in order to send it to a classifier, which requires extra time for the generation of the regional proposals; (3) Faster-RCNNs [17] implement a region proposal network (RPN) as a substitute for the selective search, and connect the ROI (region of interest) pooling layer to extract the fixed vector of each region proposal to do the regression and classification separately. Faster-RCNN detector training could be a better choice in wave power density hotspot identification in terms of saving time.

Trained with the visualized meteorological dataset to identify wave power density hotspot behavior, this paper focuses on exploring the feasibility of implementing the deep convolutional neural network, combined with energy harvesting, on wave power hotspot identification through pretrained models. The pretrained models are based on the structure of a convolutional neural network (CNN), which extracts high-level features quite efficiently. The resolution of the visualized images was in a $592 \times 1200 \times 3$ format, which was projected with geographic coordination, and which distinguished the density strength level with the customized color bar. Currently, the manual labeling of the objectives still takes the main occupancy in the preparation of deep learning training datasets. Even though some of the lab algorithms appeared for more application, they still have limitations in terms of the label efficiency and the object type. The labeling was completed manually in this research, with the MATLAB image labeler. ResNet50 was implemented to train the detector to identify wave power density hotspots, which had a good performance, higher accuracy, and a shorter computing time, when compared with MobilNetV2, InceptionV3, and Inception-ResNetV2, on 2D visualized image identification [18]. The validation dataset was chosen from the same sources, including the entire annual data for 1980, and for one week of 1998, and one week of 2017, and was never used for training or testing. The validation dataset was only used for the validation of the prechosen model. Without losing the resolution of the images, the remaining dataset was divided, $70 \%$ into a training set, and $30 \%$ into a testing set, after selecting the validation dataset. The validation dataset was labeled manually as well. The testing dataset consisted of unlabeled visualized images of wave energy densities, which were adopted to identify the trained detector performance. The accuracies and statistical performances were tested through four pretrained models, and the results are discussed in [18], with the accuracy trained by ResNet50 as $96.72 \%$.

The Gulf of Mexico was selected as the study area, where the wave power density threshold range was set up from $20 \mathrm{~kW} / \mathrm{m}$ to $65 \mathrm{~kW} / \mathrm{m}$ when visualizing the calculation results and deciding on the color bars. The detected bounding box (width, height, $x$ axis of left top point, $y$ axis of left top point) is an imaginary rectangle that contains the wave power density hotspot. A deep convolutional neural network was combined with image processing in order to identify the segmentation of the wave power density hotspot within the bounding box above the level of the threshold. The average strength, size, pixel 
coordinates, and geographic coordinates of the wave power density hotspot were saved for further analysis. Figure 3 shows an example of an identified hotspot in a bounding box.

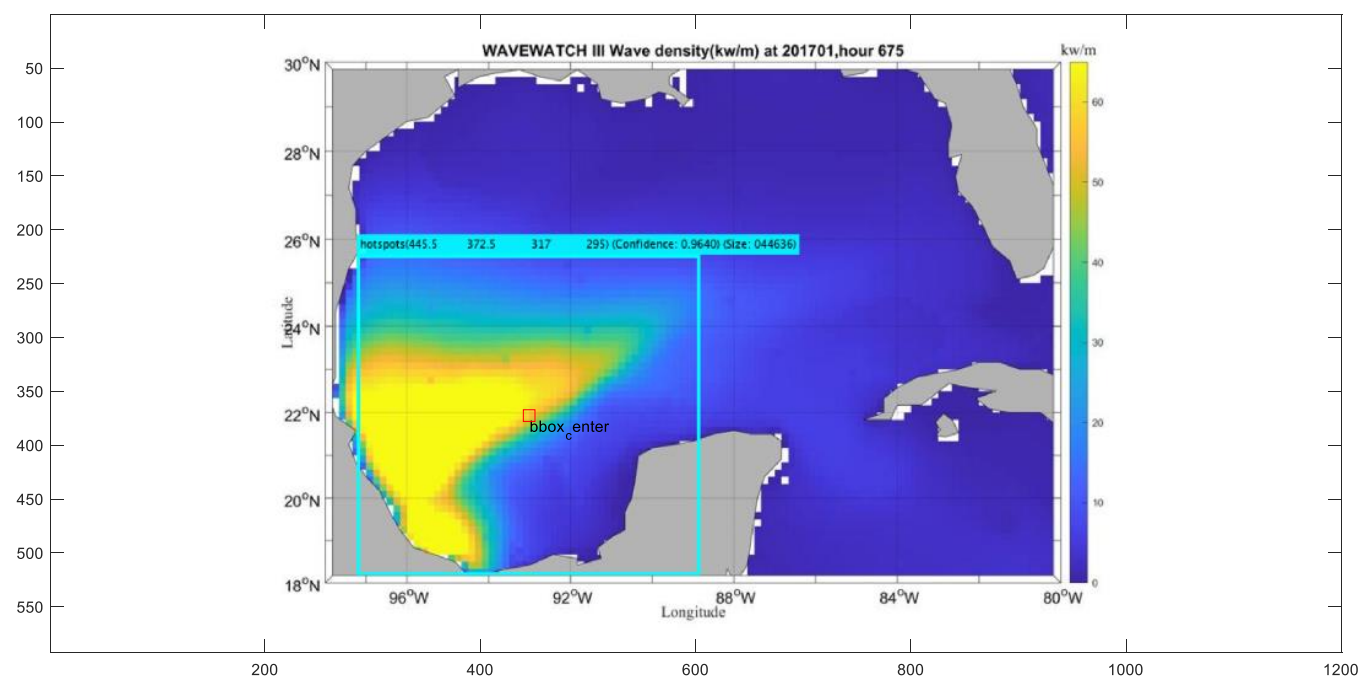

Figure 3. Validation data of the trained detector in the Gulf of Mexico at 15:00, 27 November 1988.

\subsection{Correlation Analysis}

After identifying all the hotspots and normalizing all the data, the Pearson correlation was implemented to calculate the correlation between the numbers and strengths of the sea surface temperatures and salinities of the wave power density hotspots, respectively. The Pearson correlation coefficient is an efficient mathematical way to estimate whether there is a relationship between variables:

$$
R=\frac{N \sum x_{i} y_{i}-\sum x_{i} \sum y_{i}}{\sqrt{N \sum x_{i}^{2}-\left(\sum x_{i}\right)^{2}} \sqrt{N \sum y_{i}^{2}-\left(\sum y_{i}\right)^{2}}}
$$

The $R$ value is calculated to obtain the preliminary result of the linear dependence. The $R$ value ranged between -1 and 1 . The correlation matrixes of the two variables represent the correlation coefficients for each pairwise variable combination [19]:

$$
R=\left(\begin{array}{ll}
\rho(x, x) & \rho(x, y) \\
\rho(y, x) & \rho(y, y)
\end{array}\right)
$$

When two vectors are increased/decreased in the same/opposite direction, they are strictly positively/negatively correlated. When two vectors are perpendicular, they are completely unrelated.

\section{Results}

The detailed results of the wave power hotspot distribution and its correlations with surface temperatures and salinities are discussed in this section. Through the deep convolution neural network, the wave power density hotspots over 40 years were identified and classified. The distribution of the wave power density hotspots for the 40 years was recognized and visualized by Faster-RCNN on the basis of the ResNet50 pretrained model by transfer learning. Meanwhile, the corresponding distribution of the ocean surface temperatures from 1979 to 2019 was visualized, and the correlations with the wave power density hotspot distribution, in monthly and annual averages, were revealed.

\subsection{Wave Power Hotspot Distribution}

Wave energy has the character of changing constantly, both temporally and spatially. By considering the properties of the wave power density hotspots, it is possible to obtain de- 
tailed behaviors of wave energy for efficient harvesting. In terms of the different harvesting devices applied, the wave energy density output makes a difference. In terms of a Pelamis $750 \mathrm{~kW}$ wave converter and its power curve [20-24], the available minimum wave density required to generate wave power is $18.38 \mathrm{~kW} / \mathrm{m}$ to drive the device, and $63.38 \mathrm{~kW} / \mathrm{m}$ to harvest the maximum affordable wave power of the device. In this paper, to explore the wave power density hotspot distribution, the wave power densities ranged from $20 \mathrm{~kW} / \mathrm{m}$ to $65 \mathrm{~kW} / \mathrm{m}$. The result may have slight differences, depending on different harvesting devices. Furthermore, the color bar of the visualized images was set up to comply with all of the 40-year images in order to obtain the unique correspondence. Hence, the range of the color bar was chosen as $20-65 \mathrm{~kW} / \mathrm{m}$ in order to display and cover the available values under the given threshold levels.

On the basis of the wave power density range, three thresholds were set up at $20-35 \mathrm{~kW} / \mathrm{m}, 35-50 \mathrm{~kW} / \mathrm{m}$, and $50-65 \mathrm{~kW} / \mathrm{m}$, as different wave power density hotspot strength levels, in order to explore the correlations with the ocean surface temperatures and salinities in more detail. Through deep convolutional neural network training, the detector could identify the wave power densities within the thresholds previously identified by the bounding boxes. The training dataset was labeled manually, with the wave power density active areas in the visualized images.

Since the shape of a wave power density hotspot is irregular, the bounding box generated from the deep convolutional neural network cannot track the wave energy behavior directly. The application of the coordinates of each bounding box to the representations of the locations of each hotspot was not accurate enough to indicate the wave power density strength levels. Hence, finding the highest strength of each wave power density hotspot, instead of the upright coordinates of the bounding box, became the initial step in the identification of each hotspot. The center coordinates of the bounding box may not be included within the irregular shape. Thus, it was reasonable to adopt the points with the highest wave density values to represent the highest strength levels of the entire wave power density hotspot. If there were more than one pixel point within a hotspot area with the same highest value, the nearest distance from the center coordinate point was chosen as the highest one. As the color bar implies Parula as the default when visualizing the wave power density images, it maintains the same color system as the images in order to match the colormap value with each pixel. The correspondence of the RGB index map and the colormap ( $256 \times 3$ matrix) was applied to identify and create the relationship among the wave power density values, the colormap, and the strength level of the image. The pixels of the image match the ones all over the color bar. On the other hand, the realistic area of each wave power density hotspot could be calculated by the amount of pixels, with strength levels within $20-65 \mathrm{~kW} / \mathrm{m}$. Moreover, each pixel matched the geographic coordinates in latitude and longitude to locate the hotspot.

After detecting the 40-year visualized images, 99,821 images were involved in the detection process, and 16,219 hotspots were identified between 1979 and 2019. Several scenarios exist for the hotspot distribution. For instance, each image (one image per every $3 \mathrm{~h}$ ) may include more than one hotspot during the time frame, or only one hotspot that lasts for the entire time frame. Figure 4 shows the percentages of the numbers of hotspots identified under different threshold levels. On the basis of image processing and identification, the results collected and calculated the strengths (corresponding color data), areas, geographic coordinates, pixel coordinates, and the maximum strength points (new bounding box center coordinates). The coastal line of the Gulf of Mexico was added in order to constrain all of the hotspot points within the Gulf of Mexico.

The number of hotspots was divided into three levels and dispersed on a one-degree grid, which creates a matrix of $12 \times 18$ grids in latitude and longitude. Figure 5a shows the number of detectable hotspots and their strength levels. Figure $5 \mathrm{~b}-\mathrm{d}$ displays the different levels of the hotspot strengths and amounts: $20-35 \mathrm{~kW} / \mathrm{m}, 35-50 \mathrm{~kW} / \mathrm{m}$, and $50-65 \mathrm{~kW} / \mathrm{m}$. The middle level of $35-50 \mathrm{~kW} / \mathrm{m}$ occupies the largest proportion of the total, which is scattered along the entire Gulf of Mexico, with a large proportion concentrated along with 
the Yucatan Channel. The hotspots with the high strength levels are focused along the Bay of Campeche.

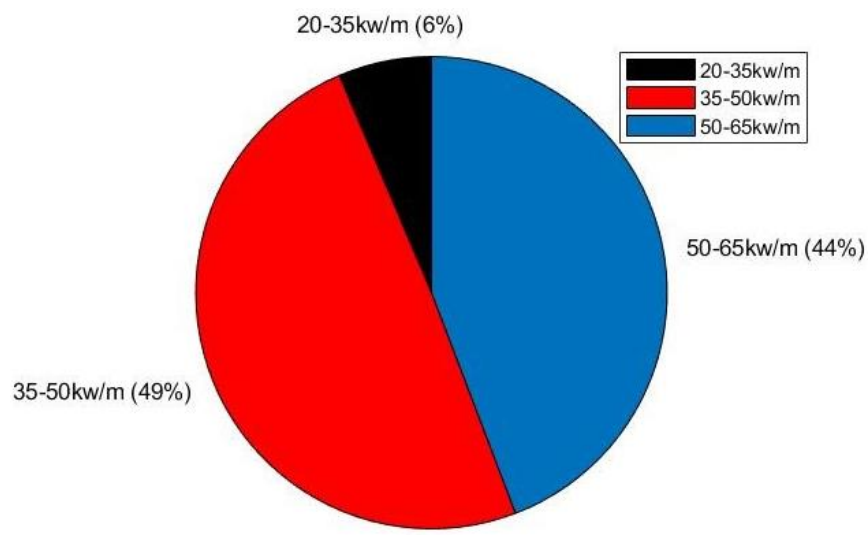

Figure 4. Wave power density hotspot distribution under different thresholds in the Gulf of Mexico (latitude $\left(18^{\circ}, 30^{\circ} \mathrm{N}\right)$, longitude $\left(-98^{\circ},-80^{\circ} \mathrm{W}\right)$ between 1979 and 2019.

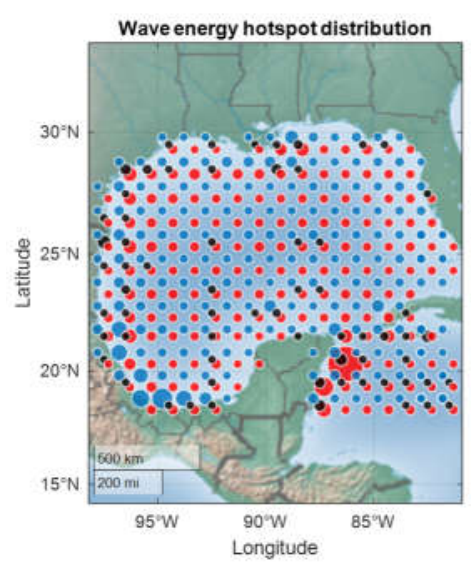

(a)

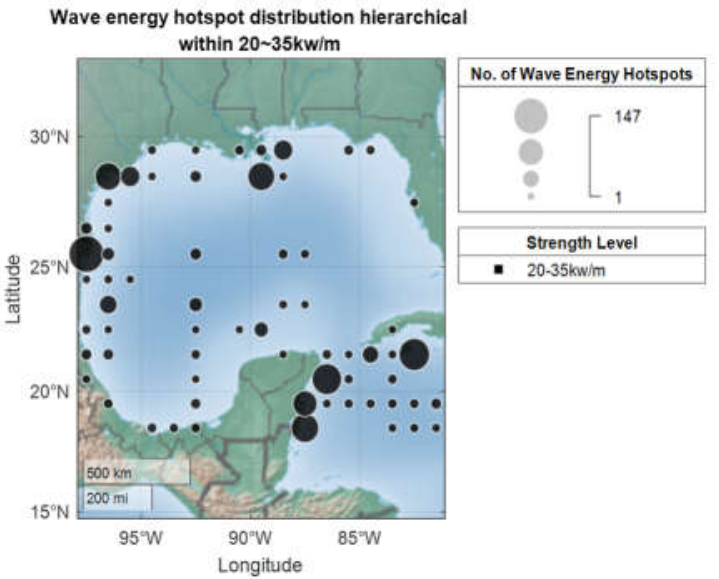

(c)

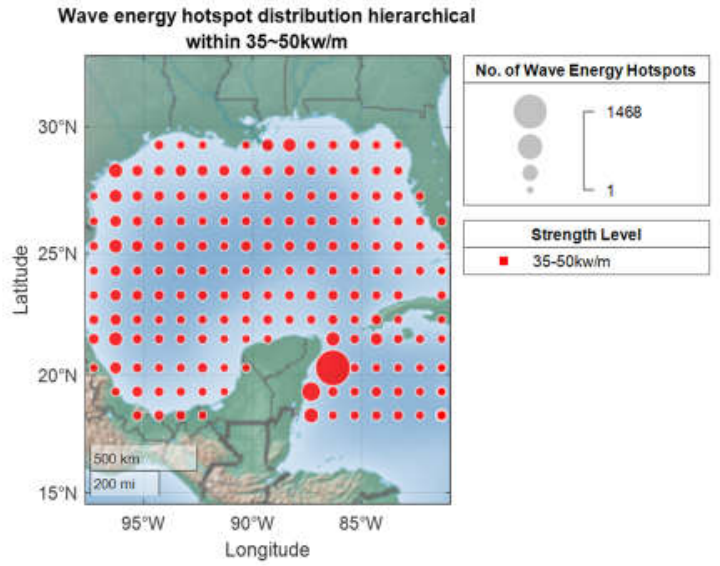

(b)

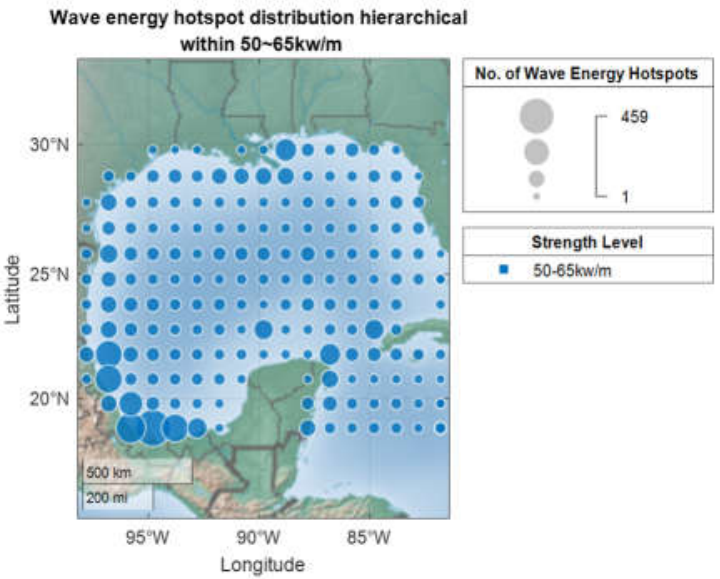

(d)

Figure 5. Wave energy density hotspot amounts and strength levels, within $1^{\circ}$ by $1^{\circ}$ grids, in (a) $20-65 \mathrm{~kW} / \mathrm{m}$; (b) $20-35 \mathrm{~kW} / \mathrm{m}$; (c) $35-50 \mathrm{~kW} / \mathrm{m}$; and (d) $50-65 \mathrm{~kW} / \mathrm{m}$, in the Gulf of Mexico (latitude $\left(18^{\circ}, 30^{\circ} \mathrm{N}\right)$, longitude $\left(-98^{\circ},-80^{\circ} \mathrm{W}\right)$.

\subsection{Correlations with Ocean Surface Temperature}

In order to find the correlations between the wave power density hotspot distribution and the ocean surface temperatures, the same time series and geographic location needed 
to be addressed. The detected hotspots are dispersed around the entire area of the Gulf of Mexico, and the temperature dataset is scattered in different resolutions of $1 / 4$ of a degree. Normalization is also a necessary process in formatting the data with different units for further analysis, which removes any anomalies complicating the analysis. The monthly values within one-degree grids were calculated for 40 years, which form a vector of all the values at the same location and in the same time series. During the identification of the neural network learning, normalizing the data ensures that different features are within the same range of values, which speeds up the gradient descents. The process removes any data with error and redundancy, which sharpens the data, making it more logical as well as easier to locate and contrast.

Figure 5a shows the correlations between the wave power density hotspot strengths and the monthly ocean sea surface temperatures (fahrenheit). The $R$ coefficient values range from -0.161 to 0.205 . The points indicate the $R$ values with matching colors in different levels. The locations of the $\mathrm{R}$ values in each grid were set up along the upright of each grid, which may have forced some of the points to be shown close to or over the coastline in the image. The highest positive $R$ values were concentrated in the Yucatan Channel, as well as in the Bay of Campeche, along the Yucatan Channel. The Gulf Stream passes the Yucatan Strait and flows along the coast of the Gulf of Mexico, and most of it then rapidly turns towards the estuary of the Florida Strait, into the Atlantic Ocean. The Gulf Stream brings a huge amount of heat, which is emitted along the Yucatan Channel flowing along the Bay of Campeche. The wave hotspot strength distribution indicates the positive correlations with the ocean surface temperatures matching the $R$ value trend (the green points in Figure 6a). The majority of the $R$ values were located between -0.1 and 0.1 , which indicates no correlations within the range of the Gulf of Mexico.

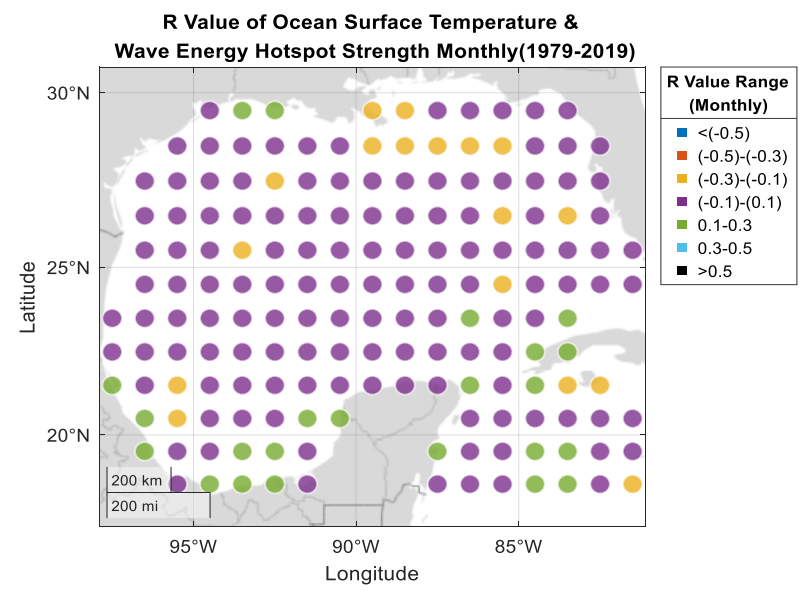

(a)

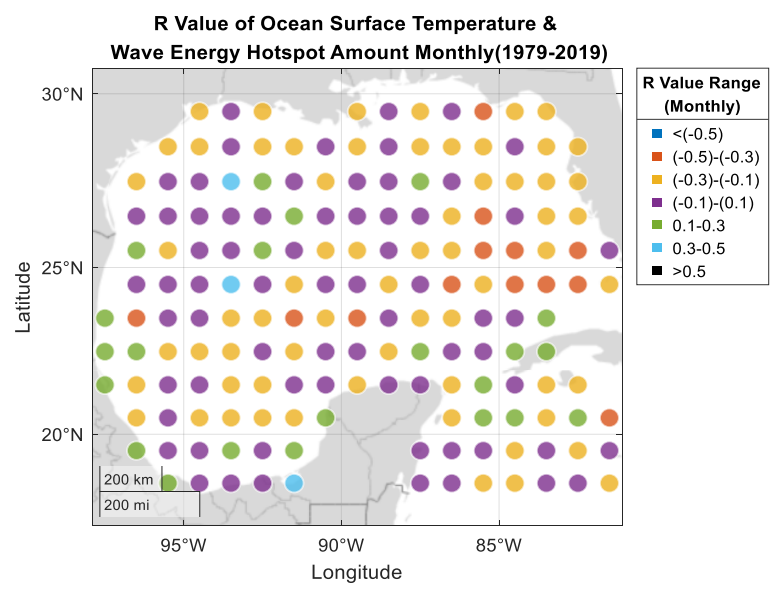

(b)

Figure 6. Correlation $R$ values of wave power density hotspots: (a) strengths (20-65 kW/m); and (b) monthly amounts and ocean surface temperatures (April 1979-May 2019), within $1^{\circ}$ by $1^{\circ}$ grids, in the Gulf of Mexico (latitude $\left(18^{\circ}, 30^{\circ} \mathrm{N}\right)$, longitude $\left(-98^{\circ},-80^{\circ} \mathrm{W}\right)$ ).

However, the $R$ values of the numbers of wave power density hotspots in the fixed grids with ocean surface temperatures provided higher correlation values, from -0.474 to 0.393 . The highest $R$ values (absolute values) increased to 0.474 , and they were concentrated in the Straits of Florida, and were scattered slightly along the Sigsbee Deep area, in orange dots, which means that the number of hotspots deceased with the ocean surface temperatures. They formed a spread branch along the Straits of Florida to the interior of the Gulf of Mexico. The majority of the numbers of hotspots spread with mild negative correlations with temperature. The maximum positive value (0.393) was higher than the strength correlations as well. The distribution of the positive dots started to scatter from the Yucatan Channel to the interior. The results imply that the strengths of the wave power density hotspot temporal and spatial distributions along the Yucatan Channel followed the 
temperature trend, with a decreasing number of hotspots in the monthly average dataset comparison and analysis.

If there were not obviously strong correlations in the monthly averages of the 40 years with the wave power density hotspot strengths, it was reasonable to explore the annual average datasets from the same period for comparison. As shown in Figure 7, the $R$ values definitely increased, either positively or negatively. The medium positive correlations increased up to 0.414 , which were mainly scattered along the estuary of the Yucatan, and along the Bay of Campeche, and which spread to the center of the study area. The negative relations occurred sharply to take the places of the locations of the purple dots (no relations $R$ values), bringing the maximum $R$ value to -0.486 in the annual average strengths.

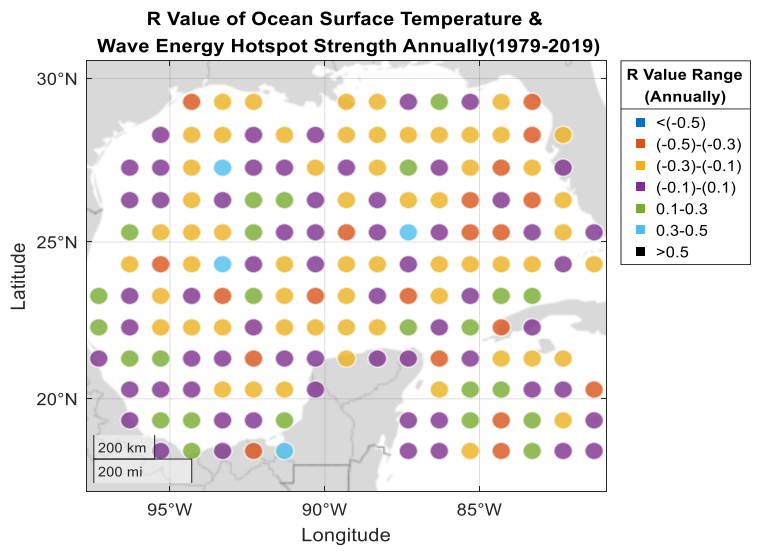

(a)

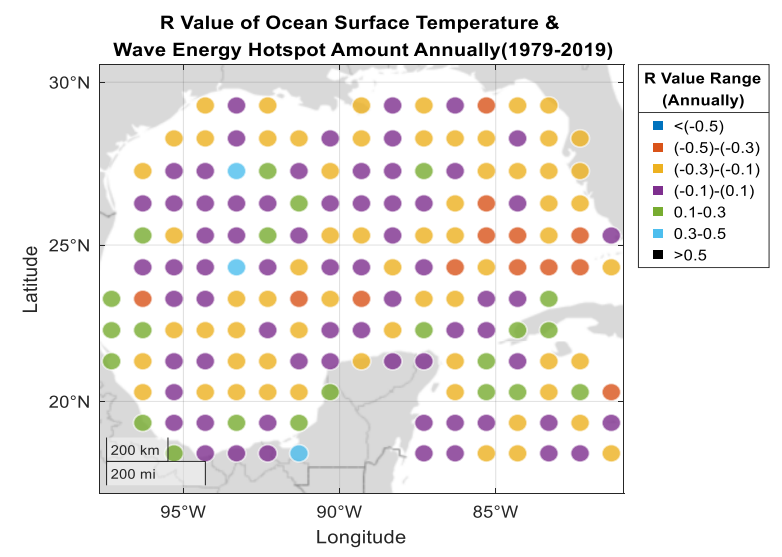

(b)

Figure 7. Correlation $R$ values of wave power density hotspots: (a) strengths (20-65 kW/m); and (b) annual amounts and ocean surface temperatures (April 1979-May 2019), within $1^{\circ}$ by $1^{\circ}$ grids, for the Gulf of Mexico (latitude $\left(18^{\circ}, 30^{\circ} \mathrm{N}\right)$, longitude $\left(-98^{\circ},-80^{\circ} \mathrm{W}\right)$.

\subsection{Correlations with Sea Surface Salinity}

The correlations between the salinities and the strengths of the wave hot spots in the temporal and spatial distributions, on monthly and annual scales, have obvious differences as shown in Figure 8. The $R$ value changed from -0.371 to -0.885 in a negative direction, and from 0.219 to 0.771 in a positive direction. The annual average strengths have a palpable tendency along with those of the western coastal area. Contrarily, the negative values are concentrated along the coastal regions of Florida from the Yucatan Channel. Compared to the numbers of hotspots in each grid, the correlations with the surface temperatures went after, with strength levels from -0.941 to 0.838 , which definitely indicates strong correlations in both directions. The salinity maximum arrived at 39.0683 PSU; higher salinities affect the density of the seawater, which governs the wave generation cycle and climate change. The monthly averages $(-0.441 \sim 0.201)$ of the salinities barely change because the salinity is predominantly driven by the concentration change that results from evaporation, precipitation, and so on. The annual variations reveal that the correlations are more distinct instead. This could be one of the parameters for determining and exploring wave energy behaviors. In particular, it could be used to identify potential wave energy harvesting sites.

Comparatively, the sea surface temperatures have relatively stronger correlations with the wave power density hotspot strengths in the annual, rather that the monthly, data. The results imply that, when the ocean surface temperature is increased by the Gulf Stream, the strengths of the temporal and spatial distributions of the wave power density hotspots along the Yucatan Channel follow the temperature trend, with a decreasing number of hotspots in the annual average dataset comparison and analysis. Obviously, the sea surface salinity had stronger correlation patterns detected with the wave power density hotspot strengths and numbers in the annual averages. The negative tendency follows the coastal regions of Florida from the Yucatan Channel, and the positive $R$ values show a tracking line 
along with the western coastal area, from the pattern. Hence, the result patterns indicate the possibility of analyzing the existing correlations with the corresponding parameters, instead of traditional data analyzation. Deep learning makes the pattern analyzation capable and achievable, as it takes advantage of the layers of the network to handle the large dataset processing, and it also solves the time-consuming issue.

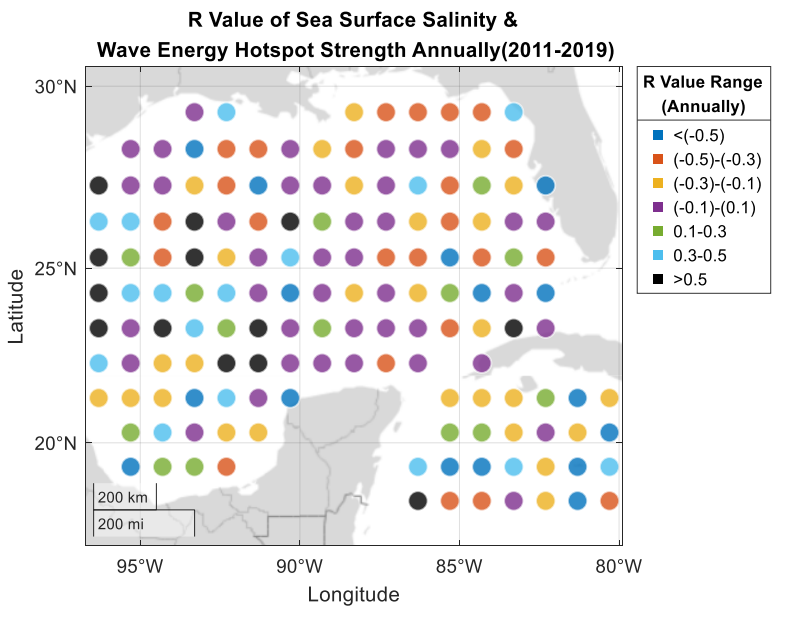

(a)

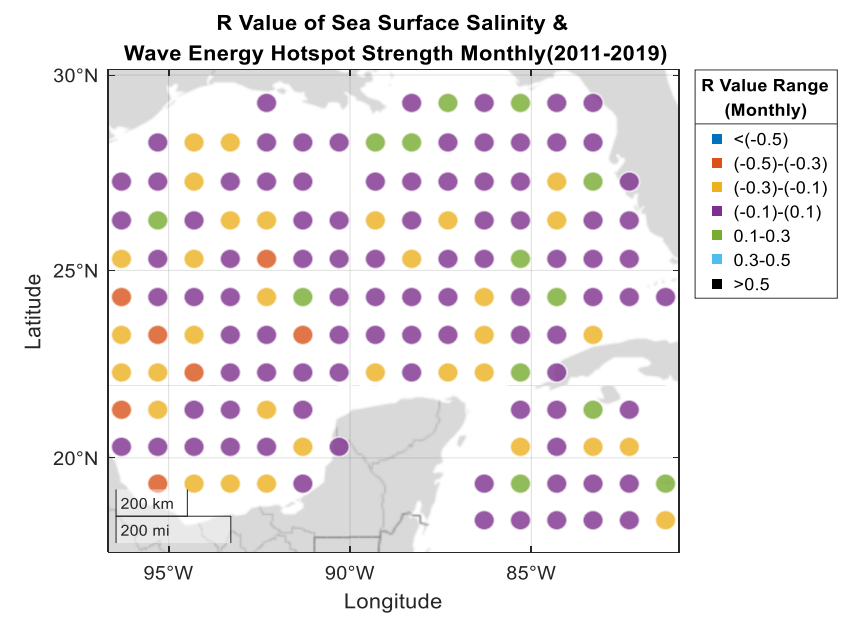

(b)

Figure 8. Correlation $R$ values of wave energy density hotspot strengths and sea surface salinities: (a) annually; and (b) monthly (January 2011-May 2019), within $1^{\circ}$ by $1^{\circ}$ grids, in the Gulf of Mexico latitude $\left(18^{\circ}, 30^{\circ} \mathrm{N}\right)$, longitude $\left(-98^{\circ},-80^{\circ} \mathrm{W}\right)$.

\section{Conclusions}

In this project, a deep convolutional neural network was implemented to classify and locate the wave power density hotspots in the Gulf of Mexico. The study area ranged between latitudes of 18 and 30 degrees, and the longitudes ranged between -98 and -80 degrees, which were divided into one-by-one-degree grids, to comply with the different resolutions of the wave energies, ocean surface temperatures, and sea surface salinities. The correlations were calculated and analyzed through visualized images, instead of through the statistical plots used in previous research. All of the wave power density hotspots over 40 years between $20-65 \mathrm{~kW} / \mathrm{m}$, were identified, collected, and visualized as images. Utilizing image processing, correlation analysis, and data analysis, 16,219 wave power density hotspots were filtered for further analyzation from 99,821 located hotspots. The wave energy forecasting model requires that many parameters be involved, and wind is definitely the mainly cause of wave generation. This paper explores the possibility of using different parameters in a comparative and indirect way in order to support, understand, and explore wave energy behaviors. After all, salinity and temperature are two of the parameters that are obtained comparatively easily, compared to the other corresponding wave parameters.

The wave power density was used to represent the wave strength in each wave hotspot. The use of wave power density hotspots offers a fresh perspective from which to look into wave energy harvesting and the device sites chosen as references. This research explores the wave power density hotspot distribution in the Gulf of Mexico over the past 40 years, and it analyzes the wave hotspot distribution as it is impacted by the physical properties of the ocean environment, either directly or indirectly. In order to understand the more detailed movements and correlations, along with the ocean surface temperature and salinity factors, the correlations of the wave density hotspot distribution were analyzed and explored for the Gulf of Mexico. Previous research has conducted analyzations on wave components, which include the significant wave heights and the dominant wave periods, with the ocean surface temperatures and salinities. However, there is no research that focuses on the wave power density distribution with the external physical factor correlations. This research 
reveals the correlation patterns between the wave power density distribution and the sea surface temperatures/salinities in quantification calculations. Even though further research on the correlation coefficient needs to be undertaken, with more details displayed, this pattern analysis offers a data analysis of wave behavior in a different way, and it could provide a hint for future research on wave energy harvesting.

As the predominant source of waves, wind plays a critical role in wave generation and behavior. Wave forecasting requires feedback on the spatial distribution and changes in the wind speed and direction on the sea in advance; thus, the accuracy is closely related to the accuracy of wind speed and direction forecasting. Thus, more related parameters could provide more forecasting constraints and limitations to the accuracy and feedback of the forecasting. However, the accuracy of a deep convolutional neutral network is closely related to the accuracy and amount of the training data used during the model training process. Generating these training data is a time-consuming and labor-intensive process because of the heavy involvement of the manual identification of the hotspots in the initial maps and images. The optimal size of the training data should be identified in future work by comparing the accuracies of the different $\mathrm{CNN}$ models trained, using different sizes of training data. By improving the training process, better accuracies would be obtained. It should also be noted that the $R$ values changed between positive and negative in several nearby locations. Considering the size of each location/grid, it is possible that these changes may be related either to human activities, such as the use of offshore oil platforms, or to natural events, such as hurricanes or tropical storms. A more detailed analysis should be conducted to reveal the underlying reasons for these observations.

Author Contributions: Conceptualization, H.L.; methodology, H.L. and C.G.; software, C.G.; validation, H.L. and C.G.; formal analysis, C.G.; investigation, C.G.; resources, H.L.; data curation, C.G.; writing-original draft preparation, C.G.; writing-review and editing, H.L.; visualization, C.G. and H.L.; supervision, H.L.; project administration, H.L.; funding acquisition, H.L. All authors have read and agreed to the published version of the manuscript.

Funding: This research received no external funding.

Institutional Review Board Statement: Not applicable.

Informed Consent Statement: Not applicable.

Data Availability Statement: Data used in this study can be found from the databases cited in the paper.

Acknowledgments: The authors are thankful for the support of Texas A\&M University, Kingsville, the National Science Foundation (award \# 1757812), the National Institute of Standards and Technology (Award \# 70NANB20H186), and the U.S. Department of Education, Title III MSEIP (Award: P120A190085).

Conflicts of Interest: The authors declare no conflict of interest.

\section{References}

1. Kilcher, L.; Michelle, F.; Michael, L. Marine Energy in the United States: An Overview of Opportunities; NREL/TP-5700-78773; National Renewable Energy Laboratory: Golden, Colorado, 2021.

2. Jacobson, P.T.; Hagerman, G.; Scott, G. Mapping and Assessment of the United States Ocean Wave Energy Resource; U.S. Department of Energy Office of Scientific and Technical Information: Washington, DC, USA, 2011. [CrossRef]

3. EIA's November 2020 Short-Term Energy Outlook. Available online: https://www.eia.gov/outlooks/steo/ (accessed on 1 December 2021).

4. Miller, C. Wave and Tidal Energy Experiments in San Francisco and Santa Cruz. 2004. Available online: https://www. outsidelands.org/wave-tidal3.php (accessed on 2 December 2021).

5. Bozzi, S.; Archetti, R.; Passoni, G. Wave electricity production in Italian offshore: A preliminary investigation. Renew. Energy 2014, 62, 407-416. [CrossRef]

6. Stoutenburg, E.; Jenkins, N.; Jacobson, M.Z. Power output variations of co-located offshore wind turbines and wave energy converters in California. Renew. Energy 2010, 35, 2781-2791. [CrossRef]

7. Wang, X.L.; Swail, V.R.; Zwiers, F.W.; Zhang, X.; Feng, Y. Detection of external influence on trends of atmospheric storminess and northern oceans wave heights. Clim. Dyn. 2009, 32, 189-203. [CrossRef] 
8. Young, I.R.; Zieger, S.; Babanin, A.V. Global trends in wind speed and wave height. Science 2011, 332, 451-455. [CrossRef] [PubMed]

9. Vinoth, J.; Young, I.R. Global estimates of extreme wind speed and wave height. J. Clim. 2010, 24, 1647-1665. [CrossRef]

10. Reguero, B.G.; Losada, I.J.; Méndez, F.J. A recent increase in global wave power as a consequence of oceanic warming. Nat. Commun. 2019, 10, 205. [CrossRef] [PubMed]

11. Vazquez-Cuervo, J.; Gomez-Valdes, J.; Bouali, M.; Miranda, L.E.; Van der Stocken, T.; Tang, W.; Gentemann, C. Using saildrones to validate satellite-derived sea surface salinity and sea surface temperature along the California/Baja Coast. Remote Sens. 2019, 11, 1964. [CrossRef]

12. Klemas, V. Remote sensing of sea surface salinity: An overview with case studies. J. Coast. Res. 2011, 27, 830-838. [CrossRef]

13. Tolman, H.L. User manual and system documentation of WAVEWATCH III TM version 3.14. Tech. Note MMAB Contrib. 2009, $276,220$.

14. Zweng, M.M.; Seidov, D.; Boyer, T.P.; Locarnini, R.A.; Garcia, H.E.; Mishonov, A.V.; Baranova, O.K.; Weathers, K.; Paver, C.R.; Smolyar, I. World Ocean Atlas 2018, Volume 2: Salinity; Mishonov, A., Ed.; NOAA Atlas NESDIS 82; U.S. Department of Commerce National Oceanic and Atmospheric Administration: Silver Spring, MD, USA, 2018; p. 50.

15. Girshick, R.; Donahue, J.; Darrell, T.; Malik, J. Rich features hierarchies for accurate object detection and semantic segmentation. In Proceedings of the IEEE Conference on Computer Vision and Pattern Recognition, Columbus, OH, USA, 23-28 June 2014; pp. 580-587.

16. He, K.; Zhang, X.; Ren, S.; Sun, J. Spatial pyramid pooling in deep Convolutional networks for visual recognition. IEEE Trans. Pattern Anal. Mach. Intell. 2015, 37, 1904-1916. [CrossRef] [PubMed]

17. Ren, S.; He, K.; Girshick, R.; Sun, J. Faster r-cnn: Towards real-time object detection with region proposal networks. Adv. Neural Inform. Proc. Syst. 2015, 28, 91-99. [CrossRef] [PubMed]

18. Gu, C.; Li, H. Wave Power Hotspots and Behaviors Identification Using Deep Convolutional Neural Networks. In IIE Annual Conference, Montreal, QC, Canada, 22 May 2021; Institute of Industrial and Systems Engineers: Peachtree Corners, GA, USA, 2021; pp. 115-120.

19. Press, W.H.; Teukolsky, S.A.; Vetterling, W.T.; Flannery, B.P. Numerical Recipes in C, 2nd ed.; Cambridge University Press: Cambridge, UK, 1992.

20. Aderinto, T.; Li, H. Review on Power Performance and Efficiency of Wave Energy Converters. Energies 2019, 12, 4329. [CrossRef]

21. Haces-Fernandez, F.; Li, H.; Jin, K. Investigation into the Possibility of Extracting Wave Energy from the Texas Coast. Int. J. Energy A Clean Environ. 2019, 20, 23-41. [CrossRef]

22. Aderinto, T.; Li, H. Ocean Wave Energy Converters: Status and Challenges. Energies 2018, 11, 1250. [CrossRef]

23. Haces-Fernandez, F.; Li, H.; Ramirez, D. Assessment of the Potential of Energy Extracted from Waves and Wind to Supply Offshore Oil Platforms Operating in the Gulf of Mexico. Energies 2018, 11, 1084. [CrossRef]

24. Haces-Fernandez, F.; Li, H.; Ramirez, D. Wave Energy Characterization and Assessment in the U.S. Gulf of Mexico, East and West Coasts with Energy Event Concept. Renew. Energy 2018, 123, 312-322. [CrossRef] 